\title{
Influencia de el Niño-Oscilación del sur (ENOS) en la ocurrencia de heladas en el altiplano peruano
}

\author{
Influence of el Niño-Southern oscillation (ENSO) in the occurrence of frost in peruvian highlands
}

\author{
Ernesto Menacho Casimiro $^{1 *}$ \& Cristina Dávila Arriaga ${ }^{1}$ \\ * Autor de correspondencia
}

\section{Resumen}

Las fluctuaciones en los patrones de circulación atmosférica global, asociados a los cambios en la temperatura superficial del mar (TSM) en el océano Pacífico, son conocidas como eventos. El Niño-Oscilación del Sur (ENOS). Estos eventos influyen en el comportamiento de la temperatura y precipitación. El objetivo principal de este trabajo fue determinar la influencia del ENOS en la ocurrencia de heladas en el Altiplano peruano mediante el análisis de la temperatura mínima del aire en el departamento de Puno. Para ello, se aplicó el análisis de componentes principales (ACP) para obtener los patrones más relevantes de la variabilidad y se utilizó el análisis de correlaciones entre los datos en formato grilla de la temperatura mínima de Puno y la TSM de la región Niño 3.4. Las dos primeras componentes principales explican el $71.9 \%$ de la variancia total de los datos (61.9\% la primera y 10\% la segunda). El primer componente, manifestó un mismo patrón en todo el departamento; mientras que el segundo componente, presentó un patrón de dipolo entre el sur y la parte centro-norte de Puno. El patrón espacial del análisis de correlación, considerando todos los datos del año, está relacionado con el segundo componente principal: existen correlaciones positivas en la parte sur y correlaciones negativas en la parte centro-norte del área de estudio. Sin embargo, esta correlación no presentó significancia estadística al $95 \%$. El análisis de correlación separando verano e invierno está relacionado con el primea componente, con valores positivos de correlación para el verano y negativos para el invierno. Por tanto, la fase positiva (negativa) del ENOS, asociada al aumento (disminución) de la TSM en la región Niño 3.4, está relacionada con una menor (mayor) ocurrencia de heladas en Altiplano peruano durante el verano (invierno) debido al aumento (disminución) de las temperaturas mínimas del aire.

Palabras clave: heladas; temperatura mínima del aire; ENOS; temperatura superficial del mar; Altiplano peruano; análisis de componentes principales; análisis de correlación.

\begin{abstract}
Fluctuations in the patterns of global atmospheric circulation, associated with changes in sea surface temperature (SST) in the Pacific Ocean, are known as El Niño-Southern Oscillation (ENSO). These events influence in the behavior of temperature and precipitation. The main objective of this work was to determine the influence of ENSO on the occurrence of frost in the Peruvian Highlands by analyzing the minimum air temperature in the department of Puno. For that, was applied principal component analysis (PCA) to obtain the most relevant patterns of variability and was used the analysis of correlations between the data minimum temperature of Puno in grid format and TSM of Niño 3.4 region. The first two principal components explained $71.9 \%$ of the total variance of the data (the first $61.9 \%$ and $10 \%$ the second). The first component shows the same pattern throughout the department while second component has a pattern of dipole between the south and the center-north of Puno. The spatial pattern of correlation analysis, considering all the data for the year is related to the second main component: there are positive correlations in the southern and negative correlations in the north central part of the study area. However this correlation no statistical significance at $95 \%$. Correlation analysis separating summer and winter is related to the first component, with positive correlation values for summer and negative values in winter. Therefore, the positive (negative) phase of ENSO associated with an increase (decrease) TSM in the Niño 3.4 region, is related to a lower (higher) occurrence of frost in the Peruvian highlands during summer (winter) due to increased (decrease) of air minimum temperatures.
\end{abstract}

Keywords: frost; minimum temperature; ENSO; sea surface temperature; Peruvian Highlands; principal component analysis; correlation analysis.

\section{Introducción}

Una de las manifestaciones de escala global más importante de la variabilidad interanual del clima es el fenómeno El
Niño Oscilación del Sur - ENOS (Servicio Nacional de Meteorología e Hidrología del Perú [SENAMHI], 2014). Es por ello que, en varias regiones del mundo, la influencia 
del ENOS ha sido estudiada por muchos investigadores con el objetivo de entender mejor sus impactos a escala local. Se sabe que el ENOS es responsable de los cambios en los patrones de circulación atmosférica que influyen en el comportamiento de variables meteorológicas como viento, temperatura, precipitación en diferentes lugares del mundo (Pabón y Montealegre, 2008).

El Perú no es ajeno a aquella influencia, más aún siendo frontera con el océano Pacífico donde inician los eventos ENOS debido a la variación de la temperatura superficial del mar. El entendimiento de los impactos del ENOS es de gran importancia en los países en vías de desarrollo como el nuestro, donde las principales actividades económicas son la agricultura y ganadería que dependen de las condiciones climáticas del entorno.

Los impactos producidos en las regiones peruanas son mayormente relacionados a la precipitación, reflejados en inundaciones y sequías que ocurren en el norte y sur del país, respectivamente; sin embargo, también existen impactos en la temperatura del aire, no solo en la región costera, sino también en la sierra y selva. Una de las situaciones más evaluadas en la sierra, debido a los daños económicos y sociales, es la frecuencia e intensidad de las heladas que son monitoreadas a través de la temperatura mínima del aire.

Es así que, en el Altiplano peruano, las heladas meteorológicas, valor de temperatura ambiente igual o menor a $0^{\circ} \mathrm{C}$ (FAO-SENAMHI, 2010) son eventos estudiados para prevenir la pérdida de cultivos y el aumento de enfermedades respiratorias en la población. En el Perú, el 21\% de la población es afectada económica y socialmente por estos eventos, en la época de inverno, debido a los impactos en los cultivos y ganado utilizados para autoconsumo. Según el informe de la Comunidad Andina (2009), cada año se tiene pérdidas en la cosecha de papa, maíz, frejol y quinua por causa del congelamiento y deshidratación de las células (Food and Agriculture Organization [FAO] y SENAMHI, 2010); así como en la reducción del rendimiento del ganado por falta de alimento fresco; puesto que un $75 \%$ del territorio está expuesto a los impactos de las heladas.

Entonces, ¿Cómo influye el ENOS en la ocurrencia de las heladas?. Es así, que el objetivo general de este trabajo fue determinar la influencia de El Niño Oscilación del Sur (ENOS) en la ocurrencia de las heladas en el Altiplano peruano mediante el análisis de la temperatura mínima del aire. Para ello, primero se evaluará el comportamiento de la temperatura mínima del aire y su correlación con la frecuencia de heladas. Seguidamente, las anomalías de la temperatura mínima serán calculadas con la finalidad de determinar los patrones de mayor relevancia de la variabilidad mediante el análisis de componentes principales (ACP). Finalmente, se realizará una correlación temporal entre datos grillados de temperatura mínima y temperatura superficial del mar (TSM) de la región 3.4 del Océano Pacífico.
Mediante esta evaluación se quiere conocer el comportamiento de las heladas durante las fases positivas y negativas del ENOS, de esta manera aumentar la investigación en este ámbito y contribuir al diseño de mejores estrategias de prevención y mitigación de los impactos originados por estos eventos meteorológicos

\section{Materiales y métodos}

\section{Materiales}

\section{Datos observados}

Una de las variables analizadas en este trabajo, es la temperatura mínima del aire que es la menor temperatura registrada en un día a una altura entre 1.25 y 2 metros por encima del nivel del suelo (Organización Mundial de Meteorología [OMM], 1996). Esta variable está directamente relacionada con las heladas ya que, al ser el menor valor de temperatura alcanzado en el día, indica la intensidad que tendría la helada meteorológica al presentarse.

Se consideraron datos diarios de temperatura mínima del aire de 10 estaciones meteorológicas distribuidas en la región del Altiplano peruano, departamento de Puno (Figura 1). El periodo de años analizados fue de 1971 2005. En la Tabla 1, se muestran las latitudes, longitudes y altitudes de las estaciones meteorológicas.

Tabla 1. Información geográfica de las estaciones meteorológicas utilizadas

\begin{tabular}{lccc}
\hline Estación & Latitud $\left({ }^{\circ}\right)$ & Longitud $\left.^{\circ}\right)$ & Altitud $(\mathrm{m})$ \\
\hline Ayaviri & $14.88^{\circ} \mathrm{S}$ & $70.59^{\circ} \mathrm{W}$ & 3920 \\
Desaguadero & $16.57^{\circ} \mathrm{S}$ & $69.04^{\circ} \mathrm{W}$ & 3860 \\
Huancane & $15.20^{\circ} \mathrm{S}$ & $69.76^{\circ} \mathrm{W}$ & 3860 \\
Huaraya Moho & $15.39^{\circ} \mathrm{S}$ & $69.49^{\circ} \mathrm{W}$ & 3890 \\
Ilave & $16.08^{\circ} \mathrm{S}$ & $69.64^{\circ} \mathrm{W}$ & 3850 \\
Juli & $16.20^{\circ} \mathrm{S}$ & $69.46^{\circ} \mathrm{W}$ & 3825 \\
Lampa & $15.36^{\circ} \mathrm{S}$ & $70.37^{\circ} \mathrm{W}$ & 3900 \\
Muñani & $14.78^{\circ} \mathrm{S}$ & $69.97^{\circ} \mathrm{W}$ & 4119 \\
Pampahuta & $15.49^{\circ} \mathrm{S}$ & $70.86^{\circ} \mathrm{W}$ & 4320 \\
Puno & $15.82^{\circ} \mathrm{S}$ & $70.02^{\circ} \mathrm{W}$ & 3840 \\
\hline
\end{tabular}

\section{Datos de reanálisis del ECMWF}

Así mismo, fueron usados dos variables de los datos de reanálisis del European Centre for Medium-Range Weather Forecasts (ECMWF): temperatura superficial del mar (TSM) y temperatura mínima del aire. La temperatura mínima del aire del ECMWF (Dee et al., 2011) corresponde a un periodo de años de 1979 - 2005 con una resolución espacial de $0.75^{\circ}$ x $0.75^{\circ}$ y para el área de estudio mostrado en la Figura 1.

Para el caso de la TSM, los datos se refieren al valor de TSM grillado con una resolución espacial de $0.75^{\circ} \mathrm{x}$ $0.75^{\circ}$ para el área Niño $3.4\left(5^{\circ} \mathrm{N}-5^{\circ} \mathrm{S}, 170^{\circ} \mathrm{W}-120^{\circ} \mathrm{W}\right)$ en un periodo de 1979 - 2005. Cabe resaltar, que los datos grillados de TSM y temperatura mínima del aire están en formato NetCDF. 


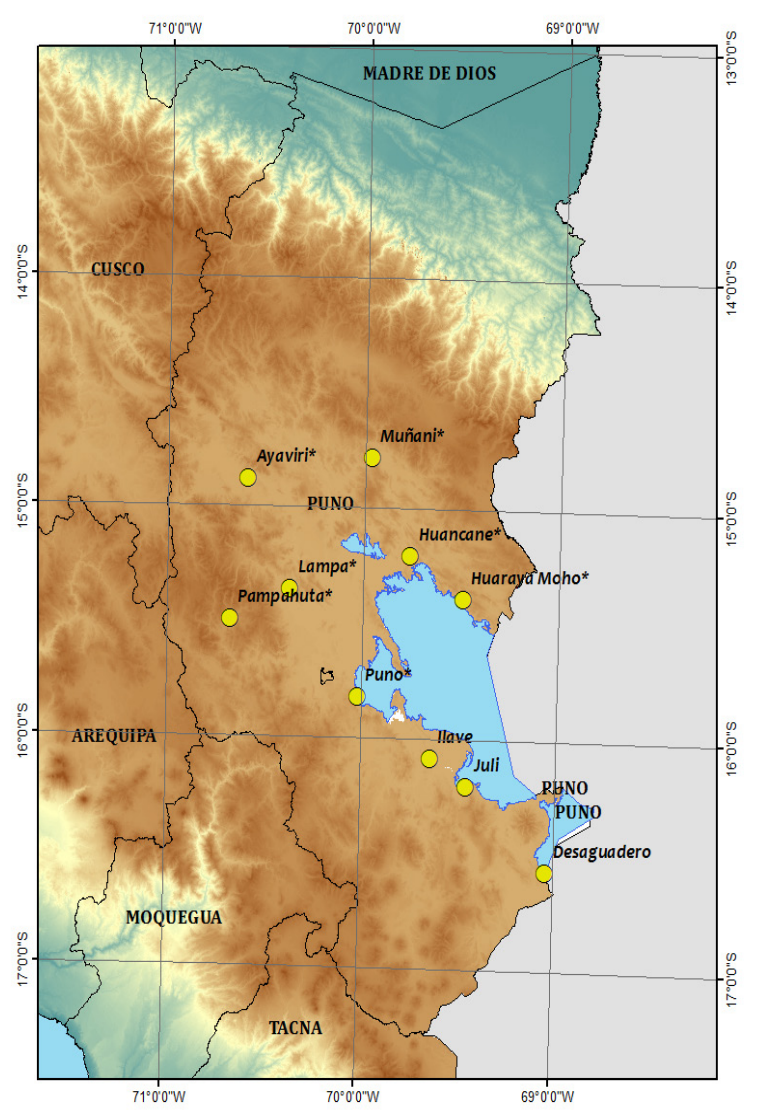

Figura 1. Mapa del área de estudio y ubicación espacial de las estaciones meteorológicas (puntos en amarillo)

\section{Métodos}

\section{Evaluación del comportamiento de la temperatura mínima del aire}

Se realizó un análisis exploratorio de los datos desde el año 1971 hasta el 2005, considerando cálculos de promedios y percentiles. En la Tabla 2, se detalla las definiciones y fórmulas de los métodos de exploración estadística utilizados en el trabajo.

Tabla 2. Métodos utilizados en el análisis exploratorio

\begin{tabular}{cl}
\hline $\begin{array}{c}\text { Método } \\
\text { exploratorio }\end{array}$ & \multicolumn{1}{c}{ Definición } \\
\hline \multirow{2}{*}{ Promedio } & $\begin{array}{l}\text { Llamado también media aritmética. Es el valor } \\
\text { característico de una serie de datos y se obtiene } \\
\text { a partir de la suma de todos los valores dividida } \\
\text { entre la cantidad de sumandos. }\end{array}$ \\
& $\begin{array}{l}\text { El percentil muestra cómo está posicionado un } \\
\text { valor con respecto al total de la muestra en una } \\
\text { serie ordenada de menor a mayor. Un ejemplo: el } \\
\text { percentil 10 es el valor bajo el cual se encuentran } \\
\text { el 10\% de los datos. }\end{array}$ \\
\hline
\end{tabular}

\section{Relación entre la temperatura mínima del aire y la frecuencia de helada}

La frecuencia de heladas consiste en contabilizar los días al mes en los que la temperatura mínima presentó valores iguales o menores a $0^{\circ} \mathrm{C}$ (Comunidad Andina, 2009). Fue calculado el promedio de la frecuencia de heladas con los datos observados para todos los meses del año de toda la serie de tiempo, así mismo se promedió la temperatura mínima mensual para los 35 años de datos. Es así, que se calculó la correlación estadística (Pearson y Spearman) entre el promedio mensual histórico de la frecuencia de helada y de la temperatura mínima del aire por cada punto de estación, con la finalidad de describir la relación existente entre las heladas y temperatura mínima del aire.

\section{Determinación de las anomalías de la temperatura mínima del aire correspondiente a los datos observados}

Las anomalías se determinaron removiendo la tendencia y la estacionalidad de cada serie de tiempo por cada punto de estación. La tendencia corresponde al cambio en el tiempo que pudiera tener la variable, ya sea de aumento o disminución, mientras que la estacionalidad está dada por la oscilación repetitiva que presenta la variable en un intervalo de tiempo que con frecuencia es anual. Como primer paso, fue removido la tendencia de la serie. Una serie temporal de datos se puede descomponer de la siguiente forma:

Donde: $T+I$

\section{$X$ : Serie temporal \\ $T$ : Tendencia temporal \\ $I$ : serie independiente de la tendencia temporal}

La T puede ser representada por la siguiente función: $T=b * t+a$, donde $a$ y $b$ son las constantes a determinar mediante el modelo de regresión lineal y $t$ es el tiempo. De esta forma, la remoción de la tendencia estaría expresado como:

$$
\mathrm{I}=X\left(b^{*} t+a\right)
$$

El segundo paso consistió en remover la estacionalidad anual de la temperatura mínima del aire. En 1807, Fourier demostró que una función continua y periódica puede ser representada por una suma infinita de ondas seno y coseno, llamados harmónicos (Carrillo, 2003). Es así, que una serie temporal puede ser representada de la siguiente forma:

$$
y_{t}=\bar{y}+\sum_{k=1}^{n / 2}\left\{C_{k} \cos \left[\frac{2 \pi k}{n}-\phi_{k}\right]\right\}=\bar{y}+\sum_{k=1}^{n / 2}\left\{A_{k} \cos \left[\frac{2 \pi k}{n}\right]+B_{k} \sin \left[\frac{2 \pi k}{n}\right]\right\}
$$


Donde:

\author{
$\mathrm{y}_{\mathrm{t}}:$ Serie temporal \\ $\bar{y}$ :Promedio de la serie temporal \\ $\mathrm{n}$ : Número de datos de la serie temporal \\ k: Número de harmónico
}

$\mathrm{C}_{\mathrm{k}}, \mathrm{A}_{\mathrm{k}}, \mathrm{B}_{\mathrm{k}}, \phi_{\mathrm{k}}$ : Coeficientes de la serie de Fourier que definen los ciclos anuales de la serie temporal.

Los coeficientes de la serie de Fourier son calculados con los siguientes criterios:

$$
\begin{aligned}
& A_{k}=\frac{2}{n} \sum_{t=1}^{n} y_{t} \cos \left(\frac{2 \pi k}{n}\right) \quad B_{k}=\frac{2}{n} \sum_{t=1}^{n} y_{t} \sin \left(\frac{2 \pi k}{n}\right) \\
& C_{k}=\left[A_{k}^{2}+B_{k}^{2}\right]^{1 / 2}
\end{aligned}
$$

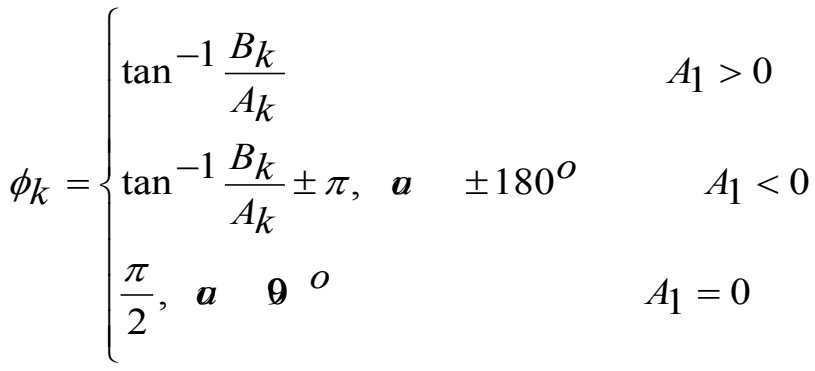

En algunos casos, el ciclo anual de la serie puede estar representado por el primer harmónico o por la suma del primero y el segundo; ello depende de la complejidad en el comportamiento de la serie en el tiempo. Una vez determinado el harmónico que representa el ciclo anual de la serie, este fue removido con una sustracción. El resultado consistió en un conjunto de series temporales sin tendencia y sin influencia del ciclo anual.

\section{Determinación de los patrones más relevantes de la temperatura mínima del aire}

A continuación, se aplicó una técnica estadística llamada análisis de componentes principales (ACP) a las anomalías de los datos observados de la temperatura mínima diaria para obtener los patrones de variabilidad más relevantes. El ACP es una técnica estadística multivariada la cual transforma linealmente un conjunto original de variables en otro conjunto de nuevas variables no correlacionadas entre sí, para poder explicar la información contenida en el primer conjunto. A ese nuevo conjunto de variables se les llama componentes principales que son combinaciones lineales de las anteriores y se van construyendo según el orden de importancia en cuanto a la variabilidad total que recogen de la muestra (Pearson, 1901).
Se tiene un conjunto inicial de variables $\left(x_{1}, x_{2}, \ldots x_{p}\right)$ y a partir de esos datos, se calcula un nuevo conjunto de variables $\left(y_{1}, y_{2}, \ldots y_{p}\right)$. Cada $y_{p}$ (donde $\left.j=1,2, \ldots, p\right)$ es una combinación lineal de las variables originales, pero no se encuentran correlacionadas entre sí:

$$
y_{j}=a_{j 1} l \mathrm{x}_{1}+a_{j 2}, \mathrm{x}_{2}+\ldots+a_{j p}, \mathrm{x}_{\mathrm{p}}=\mathrm{a}_{j}^{\prime} x
$$

Siendo:

$$
X=\left[\begin{array}{c}
x_{1} \\
\vdots \\
x_{p}
\end{array}\right]
$$

Los componentes principales vendrían a ser $a_{1}{ }^{\prime}$ de $y_{1}$, $\mathrm{a}_{2}{ }^{\prime}$ de $y_{2}$, etc. Los patrones relevantes de la variabilidad de la temperatura mínima fueron hallados a partir de las componentes principales (CP), los cuales se obtienen maximizando la variancia de la proyección. En el Anexo 1 , se explica sobre la aplicación de esta técnica estadística en la meteorología. Sin embargo, esa variancia puede ser influenciada por distintas escala de medidas o valores grandes que tendrían mayor peso en el análisis. Para el presente estudio, se consideró la matriz de correlaciones por la presencia de valores extremos en las anomalías de temperatura mínima que podrían influir en la distribución espacial de los patrones.

\section{Análisis de correlación entre anomalías de TSM del océano Pacífico y temperatura mínima del aire}

Para el caso del análisis de correlación, esta técnica se aplicó entre los campos de grilla de las anomalías calculadas de TSM región 3.4 del Pacífico y las anomalías de los datos de temperatura mínima del aire del ECMWF también en formato grilla con una resolución espacial de $0.75^{\circ} \times 0.75^{\circ}$. Se utilizó la temperatura mínima del ECMWF debido al formato grilla necesaria para la correlación con la TSM. Las anomalías corresponden a las series sin tendencia ni ciclo anual, con el objetivo de trabajar con series sin influencias estacionales. Los datos de ambas variables fueron datos diarios en el período 1979 - 2005 por la disponibilidad del sitio web del ECMWF.

El cálculo de las anomalías de ambos datos grillados fue realizado con el software Climate Data Operator (CDO) que facilita el trabajo con datos en formato NetCDF. Para calcular la correlación, tanto espacial como temporal, se utilizó el software Grid Analysis and Display System (GrADS); de esta forma se encontró patrones entre las dos variables que ayudaron a explicar las series de las componentes principales de la temperatura mínima observada en las estaciones meteorológicas.

En el análisis de correlación, se aplicó el test estadístico T-Student con un nivel de significancia de 95\% con el objetivo de comprobar si la correlación encontrada entre las variables va más allá del azar. La prueba T-Student consiste en rechazar o aceptar la hipótesis nula. Para 
ello se considera el valor de " $t$ " calculado a partir de los datos, que se compara con el valor "t" crítico de la tabla estadística correspondiente al nivel de significancia y grados de libertad (Wilks, 2006). El valor de "t" calculado está definido por la siguiente fórmula:

$$
t=\frac{r_{x y}}{\sqrt{\frac{1-r_{x y}^{2}}{N-2}}} \ldots \text { (1) }
$$

Donde:

$r_{x y}$ : coeficiente de correlación espacial hallado a partir de los datos de temperatura mínima y TSM.

$\mathrm{N}$ : número de datos

$\mathrm{t}$ : valor de " $\mathrm{t}$ " calculado

Se rechaza la hipótesis nula si el valor de " $t$ " calculado es mayor al valor " $t$ " crítico de la tabla estadística.

Para finalizar, se realizó un análisis de las intensidades de heladas para la fase positiva (El Niño) y negativa (La Niña) del evento ENOS con la finalidad de verificar si los resultados obtenidos concuerdan con la realidad.

\section{Resultados y discusión}

\section{Análisis exploratorio de los datos observados}

En la Tabla 3, se muestran los promedios y percentil $10 \mathrm{de}$ forma estacional (verano - DEF, otoño - MAM, invierno - JJA y primavera - SON) para cada punto de estación meteorológica. Vale resaltar que el percentil 10 expresa el valor de la temperatura donde el $10 \%$ de los datos de la serie es menor a dicho valor; en otras palabras, se tiene $10 \%$ de probabilidad de ocurrencia y resultan en valores atípicos, por esta razón es muy utilizado para analizar eventos extremos fríos (Wilks, 2006).

Con respecto al percentil 10, el invierno muestra valores más negativos por lo que se espera hallar temperaturas negativas más extremas. Siendo la estación de otoño el comienzo de la temporada fría, también presenta valores bajos de percentil 10 seguido de la primavera, con excepción en la estación meteorológica de Pampahuta donde en primavera se tienen valores más negativos que en otoño. Debido a la ubicación más lejana al lago Titicaca, la estación de Pampahuta muestra promedios de temperatura mínima negativos en todo el año y presentará valores más extremos que las demás estaciones meteorológicas (Tabla 3).

\section{Temperatura mínima del aire y frecuencia de heladas}

La temperatura mínima del aire está estrechamente relacionada con la frecuencia de helada, tal como se muestra en el diagrama de dispersión (Figura 2) y tabla de coeficientes de correlación de Pearson y Spearman (Tabla 4). Los promedios mensuales históricos de frecuencia de heladas y de temperatura mínima, calculados para cada punto de estación, presentan una línea de tendencia negativa; es decir los meses que poseen mayores valores de frecuencia de helada, son meses en los que la temperatura mínima es menor. Por lo tanto, existe relación inversa entre la temperatura mínima del aire y la frecuencia de heladas, siguiendo también los valores negativos de los coeficientes de correlación de Pearson y de Spearman.
Figura 2. Diagrama de dispersión entre la temperatura mínima del aire das

Tabla 4. Coeficientes de Correlación entre temperatura mínima y frecuencia de heladas

\begin{tabular}{lcc}
\hline \multicolumn{1}{c}{ Estación } & Coef. Pearson & Coef. Spearman \\
\hline Ayaviri & -0.98 & -0.99 \\
Desaguad & -0.99 & -0.99 \\
Huancane & -0.99 & -0.98 \\
Huaraya Moho & -0.99 & -0.98 \\
Ilave & -0.99 & -0.99 \\
Juli & -0.99 & -0.95 \\
Lampa & -0.98 & -0.99 \\
Muñani & -0.99 & -0.99 \\
Pampahuta & -0.96 & -0.98 \\
Puno & -0.99 & -0.99 \\
\hline
\end{tabular}

\section{Anomalías de los datos observados de temperatura mínima del aire}

La Figura 3 muestra la remoción de la tendencia (a), cálculo de los harmónicos (b) y serie temporal sin estacionalidad (c) para el punto de la estación Ayaviri. Cabe mencionar, que se replicaron estos pasos para los demás puntos de estaciones meteorológicas. La Figura 3 a presenta los datos observados originales (azul) y los datos observados sin tendencia (rojo); se puede observar que la serie no presenta una tendencia muy marcada, sin embargo es necesario eliminar esta característica para evitar su influencia en la variabilidad. En la mayoría de estaciones, al harmónico que representó el ciclo anual de la temperatura mínima fue el segundo. En la Figura 3c, se observa el comportamiento de la serie original de temperatura mínima, el ciclo anual representado por el segundo harmónico y la serie de anomalías. Las anomalías fueron obtenidas con la sustracción entre la serie original y el ciclo anual, y es justamente estas series de anomalías que se usó para en la técnica de análisis de componentes principales. 
Tabla 3. Promedios y valores del percentil 10 para cada estación meteorológica. DEF (verano), MAM (otoño), JJA (invierno) y SON (setiembre)

\begin{tabular}{ccccccccccc}
\hline & Ayaviri & Desag & Huancane & $\begin{array}{c}\text { Huaraya } \\
\text { Moho }\end{array}$ & Ilav & Juli & Lampa & Muñani & Pampah & Puno \\
\hline \multirow{5}{*}{ Promedio } & (DEF) 3.1 & 4.3 & 4.1 & 4.9 & 4.6 & 4.8 & 3.7 & 3.8 & -0.9 & 5.2 \\
& (MAM) 0.3 & 1.4 & 1.1 & 2.9 & 2.3 & 2.8 & 0.4 & 1.7 & -3.8 & 3.1 \\
& (JJA) -6.2 & -4.5 & -4.0 & -0.7 & -2.2 & -0.7 & -5.2 & -2.3 & -11.0 & -0.7 \\
& (SON) 0.4 & 1.0 & 1.7 & 3.0 & 2.1 & 3.0 & -0.2 & 1.7 & -6.2 & 3.1 \\
& (DEF) 0.8 & 1.8 & 2.0 & 3.2 & 2.8 & 2.8 & 0.9 & 1.6 & -4.2 & 3.6 \\
Percentil 10 10 & (MAM) -5.6 & -4.9 & -3.8 & -0.5 & -1.8 & -0.7 & -5.4 & -2.0 & -10.0 & -0.4 \\
& (JJA) -10.5 & -9.4 & -7.4 & -3.0 & -6.0 & -3.7 & -8.8 & -5.4 & -15.0 & -3.2 \\
& (SON) -4.0 & -4.2 & -2.0 & 0.4 & -1.2 & 0.2 & -4.8 & -1.2 & -11.6 & 0.4 \\
\hline
\end{tabular}

\section{Remoción de la Tendencia}

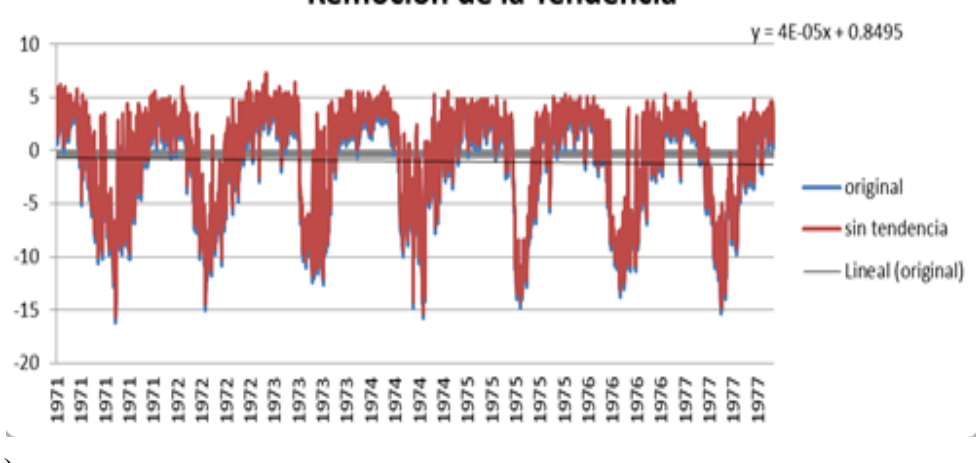

a)

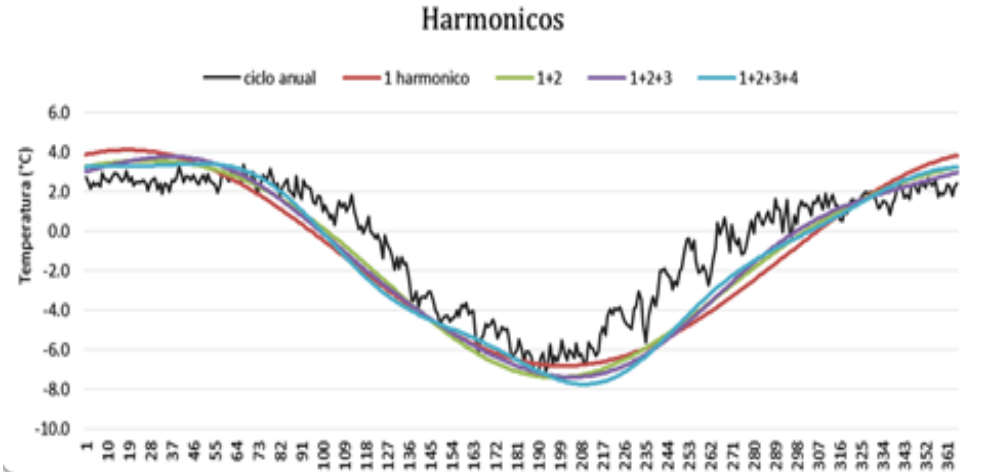

b)

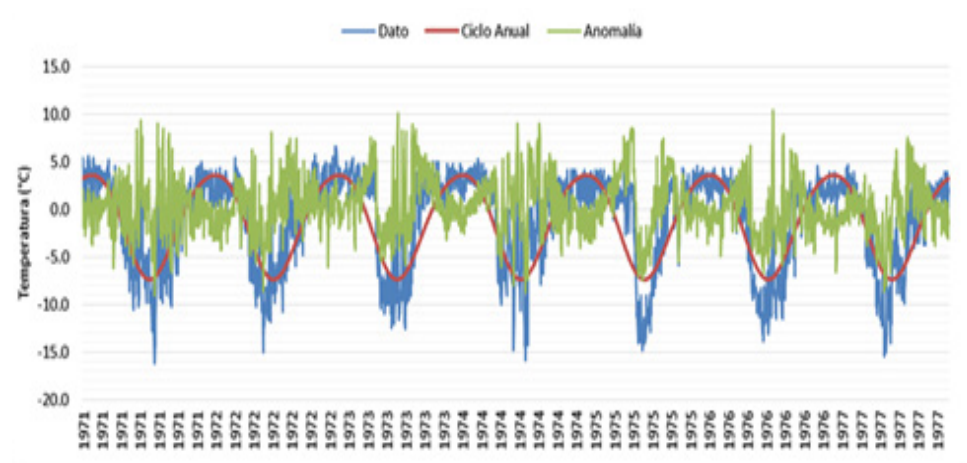

c)

Figura 3. (a) Ciclo anual de la temperatura mínima y valores de harmónicos para la estación Ayaviri. (b) Representación de la remoción del ciclo anual y anomalías como resultado para la estación de Ayaviri
Patrones relevantes de la temperatura mínima del aire

Al aplicar el análisis de componentes principales, se obtuvieron las dos primeras componentes principales encontradas explican el $71.9 \%$ de la variancia total de los datos (61.9\% y $10.0 \%$, respectivamente). En la Figura $4 a$, se observa que para la primera componente principal toda la región de estudio presenta un mismo comportamiento de la temperatura mínima. Mientras que la segunda componente (Figura 4b), se aprecia señales opuestas entre la parte norte y centro (Ayaviri, Muñani, Huancane y HuarayaMoho) y sur del área de estudio.

\section{Análisis de correlación entre TSM y temperatura mínima del aire}

En la Figura 5a y Figura 5b, se presentan los mapas del análisis de correlación entre la TSM de la región Niño 3.4 y las temperaturas mínimas para el verano e invierno, respectivamente. En ambos mapas, se aprecia un mismo patrón de correlaciones en todo Puno, con la diferencia que para el verano se muestran correlaciones positivas (Figura 5a) y para el invierno correlaciones negativas (Figura 5b). En este caso, estos patrones son muy similares al patrón de la primera componente principal que explica un $61.9 \%$ de la variabilidad de los datos.

Se analizaron algunos estudios de caso para corroborar los resultados obtenidos. En los estudios de caso, se compararon las intensidades de heladas durante los meses de verano e invierno en el año inicial y final de los eventos ENOS. Se comenzó con los eventos de fase positiva (El Niño) en los meses de verano. En la Figura 6, se presenta los mapas de anomalía de TSM en el océano Pacífico para los meses de verano del año 1982 y verano de 1983 elaborados por SENAMHI. Se puede observar que en el verano del año inicial del evento ENOS 
a)

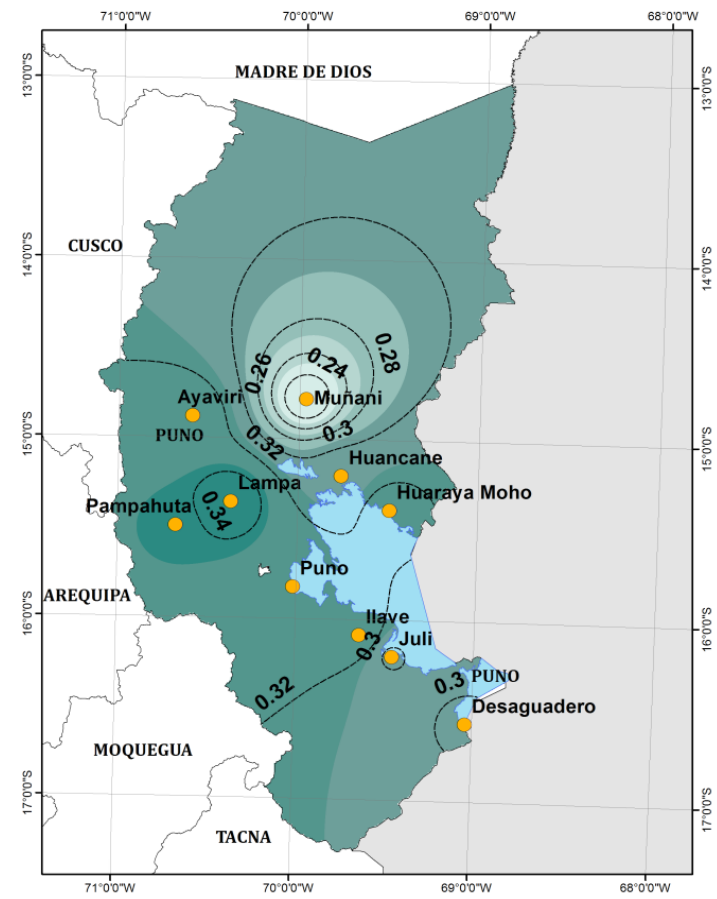

b)

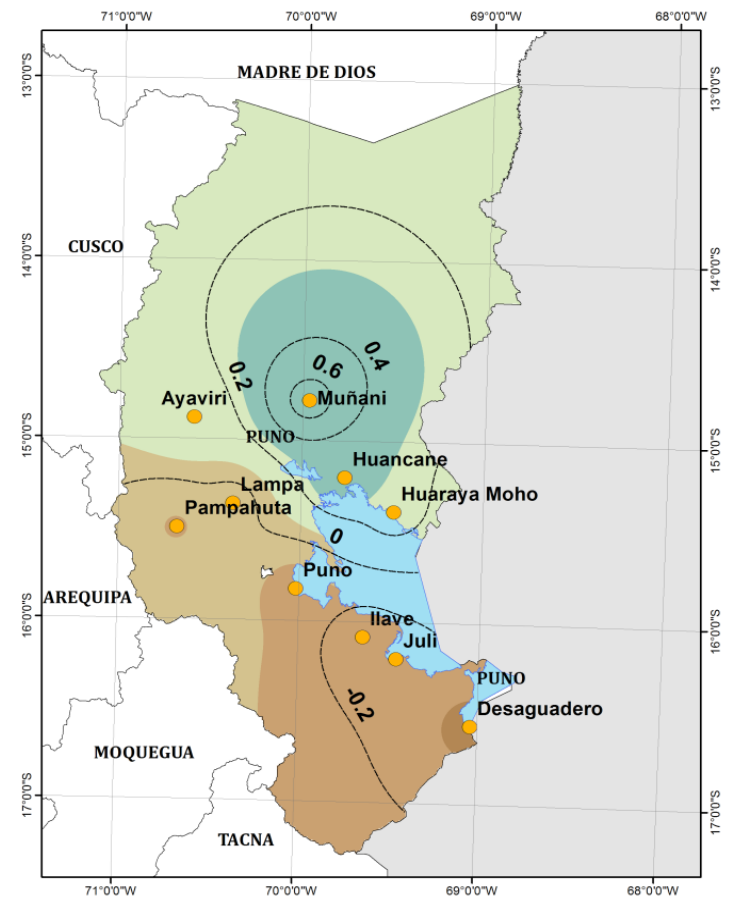

Figura 4. (a) Primera componente principal (CP1). (b) Segunda componente principal (CP2). Los valores de los contornos representan los valores de los autovectores

a)

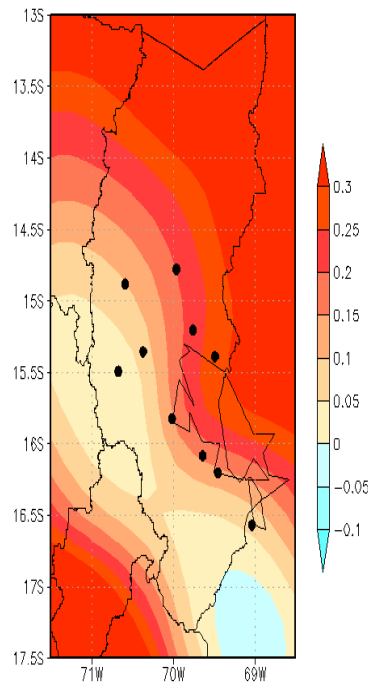

b)

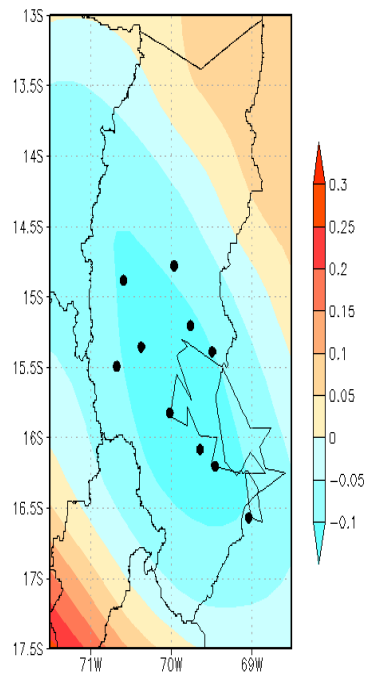

Figura 5. Patrón de correlación entre la temperatura mínima del aire en Puno y la TSM de la región Niño 3.4 para el periodo 1979 - 2005 considerando meses de verano (a) y meses de invierno (b)

(1982) la temperatura superficial del mar no presenta un fuerte calentamiento sobre la región Niño 3.4, mientras que en el verano del siguiente año (1983) se muestran anomalías positivas de hasta $5^{\circ} \mathrm{C}$. Considerando este comportamiento diferenciado de la TSM en los veranos del primer y segundo año, se presentan en la Figura 8 los gráficos de la comparación: las barras azules corresponden a la intensidad de heladas del año inicial del evento ENOS, las barras verdes al segundo año del evento ENOS y la línea roja representa los valores normales de la intensidad 
de heladas para el período de estudio. La intensidad de heladas en los meses de verano del año 1983 ( $2^{\circ}$ año), donde se presenta aumento de la TSM en la región Niño 3.4, tiene menores valores comparado con la intensidad de heladas del verano 1982 ( $1^{\circ}$ año), donde la TSM presenta valores normales en la región Niño 3.4; es decir la temperatura mínima del aire experimenta un aumento en los valores diarios (Figura 8a). Este comportamiento coincide con los resultados obtenidos en el análisis de correlación, puesto que, para el verano existe correlación positiva entre la temperatura mínima del aire en Puno y la TSM en el Pacífico central (Figura 5a). Es así que, al presentarse un aumento de temperaturas en la región Niño 3.4, las temperaturas mínimas del aire en el Altiplano también aumentarían y con ello, la intensidad de heladas sería menor durante el verano. Para el caso de los meses de invierno en la fase positiva de ENOS, la Figura 7 presenta los mapas de anomalía de TSM en el océano Pacífico durante esa estación para el año inicial 1982 y año final 1983 elaborados por SENAMHI. Durante junio, julio y agosto de 1982, la TSM muestra anomalías positivas, es decir un aumento en sus valores; mientras que en el invierno de 1983, las aguas del Pacífico central experimentan una disminución en sus temperaturas expresada en anomalías negativas.

Según el análisis de correlación para el invierno, existe correlación negativa entre la TSM de la región Niño 3.4 y las temperaturas mínimas del aire en el Altiplano. Este patrón puede visualizarse en la Figura 8b: las intensidades de heladas en 1982 ( $1^{\circ}$ año), donde la TSM aumenta, son mayores debido a una disminución de las temperaturas mínimas en la región y las intensidades de heladas en 1983 ( $2^{\circ}$ año), donde la TSM disminuye, son menores debido a un aumento de las temperaturas mínimas del aire.

\section{Conclusiones}

En el análisis exploratorio se observó que en promedio, se pueden presentar valores de temperatura mínima del aire de hasta $-11.0^{\circ} \mathrm{C}$ en los meses de invierno y de $-0.9^{\circ} \mathrm{C}$ en verano. Los valores del percentil 10 en Puno muestran que en los meses de invierno se presentan las temperaturas mínimas más extremas, con mayor énfasis en la localidad de Pampahuta.

La correlación entre la temperatura mínima y las heladas mostró que existe una relación inversa entre las dos variables; es decir, a medida que la temperatura mínima disminuye, la frecuencia de heladas aumenta.

El ciclo anual en la mayoría de estaciones meteorológicas fue determinado por el segundo harmónico.

De todas las componentes principales calculadas en el ACP, las dos primeras explican el $71.9 \%$ de la variancia total de los datos (61.9\% y 10\%, respectivamente). La primera componente presenta un patrón espacial único para todo el departamento; mientras que la segunda componente muestra un patrón espacial de dipolo, es decir la parte sur tiene una variabilidad distinta a la parte central y norte de Puno.

El análisis de correlaciones entre la región Niño 3.4 de la TSM y la temperatura mínima del aire en Puno para el periodo 1979 - 2005 presentó mayor significancia considerando por separado los meses de verano e invierno. Se obtuvo un patrón similar a la primera componente del ACP para ambas estaciones del año, siendo que un aumento/disminución en las anomalías de la TSM en la región Niño 3.4 del Oceano Pacífico induce a un aumento/ disminución de la temperatura mínima en Puno durante los meses de verano/invierno. Por ende, en la fase positiva/ negativa de los eventos ENOS, se presenta una menor/ mayor ocurrencia de heladas en el departamento durante el verano/invierno. 
SENAMHI/DGM/DMS

Reynolds - ANOMALIA DE VIENTOS (m/s) Y DE LA TEMPERATURA SUPERFICIAL DEL MAR $\left({ }^{\circ} \mathrm{C}\right)$ - CLIMATOLOGIA $(1981$ - 2010)

Diciembre de 1981

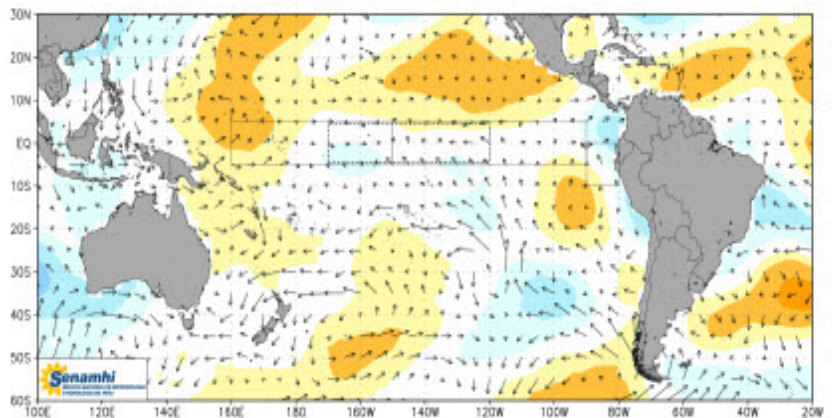

Diciembre de 1982

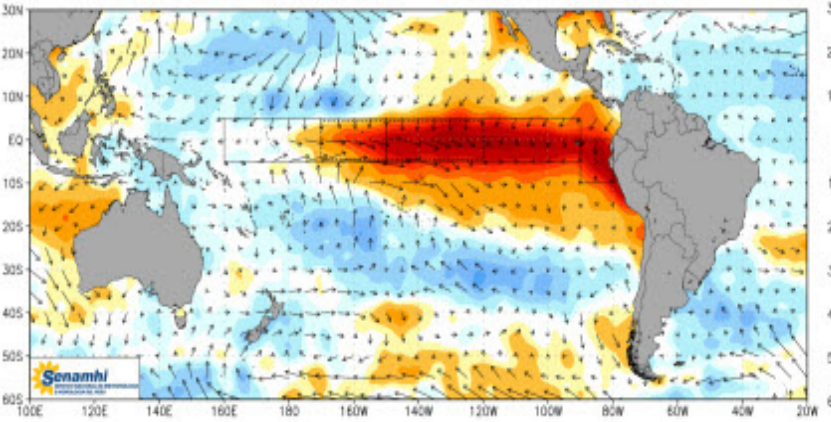

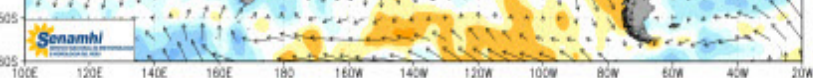

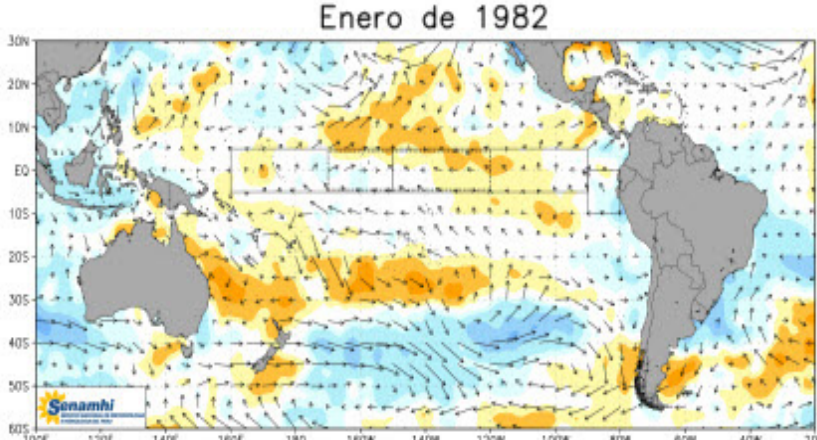

Enero de 1983

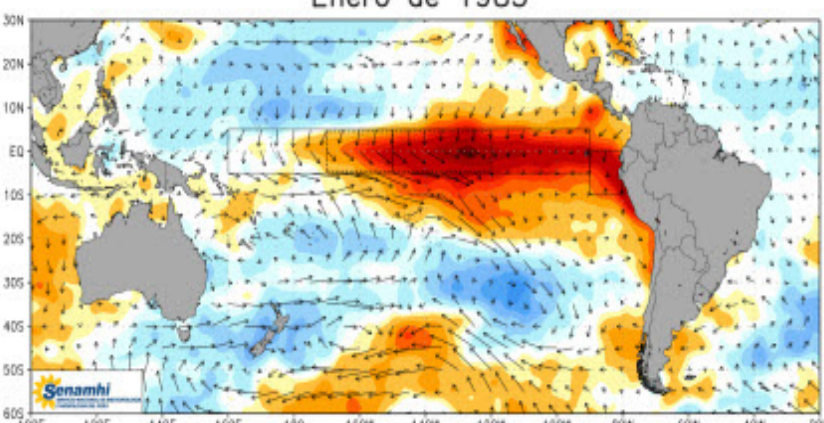

(1)

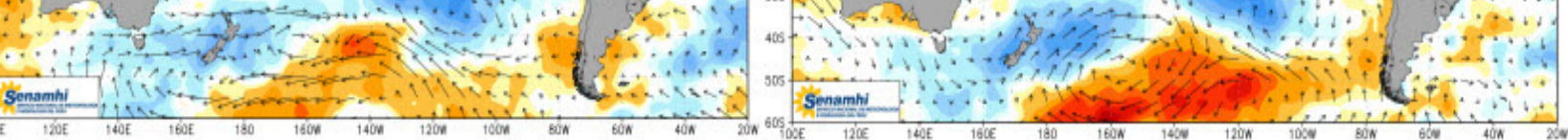

$\begin{array}{llllllllllllllllll}-5 & -4 & -3 & -2.5 & -2 & -1.5 & -1 & -0.5 & -0.2 & 0.2 & 0.5 & 1 & 1.5 & 2 & 2.5 & 3 & 4 & 5\end{array}$

Figura 6. Mapas de anomalía de la temperatura superficial del mar (TSM) sobre el océano Pacífico para los meses de verano del año 1982 (arriba) y 1983 (debajo).

Fuente: SENAMHI 
SENAMHI/DGM/DMS

Reynolds - ANOMALIA DE VIENTOS (m/s) Y DE LA TEMPERATURA SUPERFICIAL DEL MAR $\left({ }^{\circ} \mathrm{C}\right)$ - CLIMATOLOGIA $(1981$ - 2010)
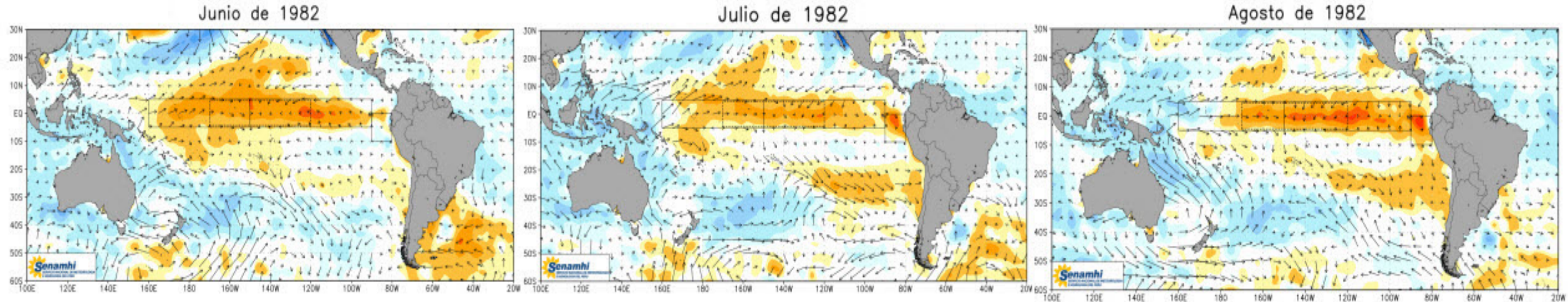

Junio de 1983

Julio de 1983
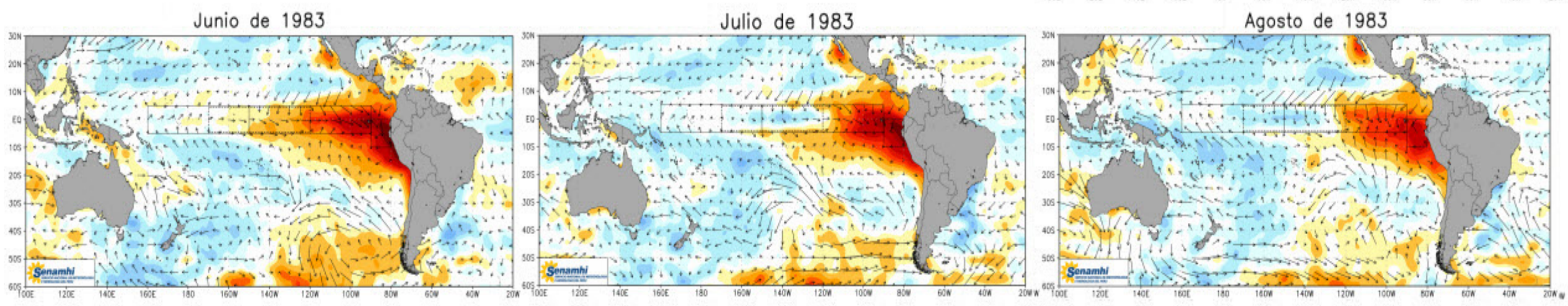

Agosto de 1983

$\begin{array}{llllllllllllllllll}-5 & -4 & -3 & -2.5 & -2 & -1.5 & -1 & -0.5 & -0.2 & 0.2 & 0.5 & 1 & 1.5 & 2 & 2.5 & 3 & 4 & 5\end{array}$

Figura 7. Mapas de anomalía de la temperatura superficial del mar (TSM) sobre el océano Pacífico para los meses de invierno del año 1982 (arriba) y 1983 (debajo).

Fuente: SENAMHI 


\section{Literatura citada}

Carrillo, C. 2003. Fundamentos del Análisis de Fourier. Departamento de Enxeñería Eléctrica Escola Técnica Superior de Enxeñeiros Industriáis. Universidade de Vigo.

Comunidad Andina. 2009. Atlas de las dinámicas del territorio Andino: Población y bienes expuestos a amenazas naturales. Capítulo: El Niño y La Niña, 150155.

Dee, D. P.; Uppala, S. M.; Simmons, A. J.; Berrisford, P.; Poli, P.; Kobayashi, S. And Others. 2011. The ERAInterim reanalysis: Configuration and performance of the data assimilation system. Quarterly Journal of the Royal Meteorological Society,137: 553-597. http:// doi.org/10.1002/qj.828

Food and Agriculture Organization [FAO] y Servicio Nacional de Meteorología e Hidrología del Perú [SENAMHI]. 2010. Atlas de heladas del Perú. p. 99.

Organización Mundial de Meteorología [OMM]. 1996. Guia de Instrumentos y Métodos de Observación Meteorológicos. Sexta edición, OMM N 8. Ginebra, Suiza.

Pabón, D. y Montealegre, E. 2008. El Ciclo El Niño, La Nina - Oscilación del Sur. Universidad Nacional de Colombia. Bogota, Colombia.

Pearson, K. 1901. On lines and planes of closest fit to systems of points in space. Philosophical Magazine 2(11):559-572.

Servicio Nacional de Meteorología e Hidrología del Perú [SENAMHI]. 2014. El Fenómeno El Niño en el Perú.

Wilks, D. S. 2006. Statistical Methods in the Atmospheric Sciences. Second edition. International Geophysics Series. 